\title{
On the Use of Local and Global Search Paradigms for Computer-Aided Diagnosis of Breast Cancer
}

\author{
$\underline{\text { A. Abroudi }}^{\text {a }}$, M. Shokouhifar ${ }^{b}$ and S. Samarasinghe ${ }^{a}$ \\ ${ }^{a}$ Integrated Systems Modelling Group, Department of Informatics and Enabling Technologies, \\ Lincoln University, Canterbury, New Zealand \\ ${ }^{b}$ Department of Electrical and Computer Engineering, Shahid Beheshti University G.C., Tehran, Iran \\ Email: Ali.Abroudi@LincolnUni.ac.nz
}

\begin{abstract}
Cancer is one of the most dangerous diseases around the world and the most common cancer among women is breast cancer. Although not all the cancer types are curable upon diagnosis, breast cancer can be cured if it is diagnosed early. The most reliable way of diagnosing breast cancer is mammographic screening which can diagnose the disease 1.5 to 4 years before it is clinically diagnosed. Double Reading is the important diagnostic process in which two experts/radiologists should read the same mammogram image to make an accurate diagnosis. But this process is not a cost-effective approach for early detection of breast cancer. Computer-Aided Diagnosis (CAD) can act as the second expert and therefore one expert would be enough for breast cancer diagnosis. In this study, we use the data extracted from low-resolution as well as high resolution mammography images. The attributes extracted from mammographic images are imported into Support Vector Machine (SVM) to classify the patients. An important point about the attributes is that sometimes there may be some irrelevant or even noisy attributes that have negative effect on the classification accuracy. Therefore, the main objective of this study is to apply local and global search paradigms in order to find the best subset of attributes to construct the most accurate $\mathrm{CAD}$ system that can effectively distinguish between benign and healthy patients. Artificial Bee Colony (ABC) is a population-based swarm intelligence algorithm with good global exploration ability, and Simulated Annealing (SA) is a robust local-search algorithm. Thus, we utilize a hybrid global and local search algorithm (named ABCSA) to simultaneously benefit from the advantages of both $\mathrm{ABC}$ and $\mathrm{SA}$. In this approach, $\mathrm{ABC}$ is firstly performed for the global exploration in the search space. Then, SA is utilized to search locally in the vicinity of the best solution found via $A B C$, in order to improve the quality of the final solution. Obtained simulation results over four different mammographic datasets show that the proposed algorithm outperforms the existing metaheuristic feature selection approaches in terms of minimizing the number of features, while maximizing the detection accuracy.
\end{abstract}

Keywords: Early detection of breast cancer, mammographic data, Support Vector Machine (SVM), feature selection, Artificial Bee Colony (ABC), Simulated Annealing (SA) 


\section{INTRODUCTION}

Breast cancer is the most common cancer among women, with around 1.7 million and 52000 estimated diagnosed cases and deaths in 2012, respectively (Torre et al., 2015). The patient can be survived if the disease is diagnosed early. Mammography, which is a low-dose X-ray image, is currently the most common way to early diagnosis of breast cancer. There are two kinds of mammography image: Screen-Film Mammography (SFM) and Full-Field digital Mammography (FFDM). In the first type, the image takes a form of photographic film, while in the latter, an electronic image is recorded directly on a computer (Faridah, 2008). Due to SFM drawbacks, such as X-ray exposure range, image contrast, and slow processing, FFDM with advantages, such as wider range, better contrast, less noise, higher quality of image, and lower dose of X-ray, has become more popular. Although it has been reported that there is little difference between overall accuracy of SFM and FFDM (Pisano et al., 2005), FFDM outperforms in particular cases (Spurgeon, 2005). As a case in point, it has been shown that FFDM is able to detect more lesions than SFM, particularly for microcalcifications (Spurgeon, 2005). It has been also found that FFDM has better accuracy for premenopausal/perimenopausal women (under 50 years old) who have denser breasts (Pisano et al., 2008).

In order to increase the reliability of mammography-based diagnosis, two radiologists are usually asked to check the mammogram images (this process is called double reading). Besides the possibility of failure in identifying lesions by radiologists, the double reading process is costly. Thus, Computer-Aided Detection (CAD) systems, which utilize computer-based algorithms to detect lesions in mammographic images and then classify patients as benign or malignant, can replace one of the radiologists to overcome the aforementioned issues (Sampat et al., 2005). Therefore, CAD system is considered as a second opinion. It has been reported that the detection sensitivity can be improved by more than $20 \%$ if a radiologist uses CAD system for breast cancer diagnosis (Brem et al., 2003). A typical CAD system extracts attributes from mammography images (through image processing techniques), and selects the most important features (through attribute selection methods), and finally classifies patients using a classification algorithm.

Four different types of attributes can be extracted from mammography images. The first group comprises clinical attributes, such as breast density, microcalcification, architectural distortion, etc. The second and third groups are calculated from the pixel grey-level intensity of the patch using statistical calculations. The second group is intensity-based descriptors (such as Mean, standard deviation, Skewness, etc.) while the third group is texture-based descriptors (such as energy, contrast, entropy, etc.). The last type of attributes includes location and shape-based features, such as area, centre of mass, solidity, etc. Using all the aforementioned attributes may not result in the most accurate CAD system because there may be some irrelevant or noisy attributes that hinder the system's ability to distinguish between healthy and cancer patients correctly. Therefore, it is crucial to adopt an appropriate attribute selection strategy in order to select the most important subset of attributes that can be later used to classify patients accurately (Abroudi et al., 2013). Numerous CAD systems for early detection of breast cancer have been presented and they have used different data as well as different metrics to measure the system classification performance (Table 1). One of the most common measures is the area under the Receiver Operating Characteristics (ROC) curve which is mostly depicted as Az. The specificity (the ratio of true negatives to healthy cases), sensitivity (the ratio of true positives to cancer cases), and accuracy (the ratio of all true classified instances to all instances) are three other measures. A list of some CAD systems applied on different mammography-based datasets is presented in Table 1. It can be seen that the number of mammogram images varies between different studies (40 to 3369) and the applied classification algorithms are also different, such as various Artificial Neural Network (ANN) extensions, Linear Discriminant Analysis (LDA), Hybrid techniques, Support Vector Machine (SVM). Therefore, it is hard to make a fair comparison between performance of different CAD systems.

Table 1. Details of some current mammography-based CAD systems

\begin{tabular}{|c|c|c|c|}
\hline Author(s) & Images & Method & Performance \\
\hline (Chan et al., 1995) & 168 & Linear Discriminant Analysis (LDA) & $A_{Z}: 0.82$ \\
\hline (Sahiner et al., 1996) & 168 & Convolution Neural Network (CNN) & $A_{Z}: 0.87$ \\
\hline (Kinoshita et al., 1998) & 92 & Back Propagation Neural Network (BPNN) & Acc: 0.81 \\
\hline (Hadjiiski et al., 1999) & 348 & Hybrid (Art2 and LDA) & $A_{Z}: 0.81$ \\
\hline (Bruce \& Adhami, 1999) & 60 & A simple Euclidian Metric & $A_{Z}: 0.9$ \\
\hline (Mudigonda et al., 2001) & 56 & LDA & Acc: 0.889 \\
\hline (Verma \& Zakos, 2001) & 58 & BPNN & $A_{Z}: 0.9$ \\
\hline (Baeg \& Kehtarnavaz, 2002) & 404 & ANN-MLP & $A_{Z}: 0.79$ \\
\hline (De Santo et al., 2003) & 102 & ANN-MLP & Sens: 0.80 \\
\hline (Campanini et al., 2004) & 512 & Support Vector Machine (SVM) & Acc: 0.725 \\
\hline (Guo et al., 2005) & 40 & Radial-Base Function (RBF) Neural Network, SVM & Sens: $0.80, A_{Z}: 0.783$ \\
\hline (Bellotti et al., 2006) & 3369 & ANN-MLP & $A_{Z}: 0.77$ \\
\hline (Timp et al., 2007) & 465 & SVM & Sens: 0.916 Spec: 0.841 \\
\hline (Al Mutaz et al., 2011) & 120 & Artificial Neural Network (ANN)-Multi-Layer Perceptron (MLP) & Sens: 0.96 Spec: 0.97 \\
\hline (Dinesh, 2011) & 190 & SVM & \\
\hline
\end{tabular}


In this paper, using both SFM and FFDM data, we aim to enhance the CAD system performance for early detection of breast cancer through a combination of heuristic local and global search methods (to find the best subset of attributes) that can render the highest accuracy for SVM classifier. The rest of the paper is organized as follows: In Section 2, SFM and FFDM datasets are described. The proposed ABCSA feature selection algorithm is illustrated in detail in Section 3. Simulation results and comparison with existing algorithms are summarized in Section 4. Finally, concluding remarks are presented in Section 5.

\section{SFM AND FFDM DATASETS}

This study uses two SFM and two FFDM datasets retrieved from Breast Cancer Digital Repository (BCDR) database (http://bcdr.inegi.up.pt) (López et al., 2012). The details of these datasets can be seen in Table2.

Table 2. Details of SFM and FFDM datasets

\begin{tabular}{|c|c|c|c|c|}
\hline Dataset Name & Dataset Type & \# Women & \# Lesions & \# Segmentations \\
\hline SFM1 & SFM & 190 & 200 & 362 \\
\hline SFM2 & SFM & 98 & 103 & 188 \\
\hline FFDM1 & FFDM & 64 & 79 & 143 \\
\hline FFDM2 & FFDM & 162 & 230 & 455 \\
\hline
\end{tabular}

The list of attributes (including clinical, intensity-, Texture-, location and Shape-based features) and a short description of each are summarized in Table 3 (Moura \& López, 2013; Moura et al., 2012).

Table 3. The list of attributes

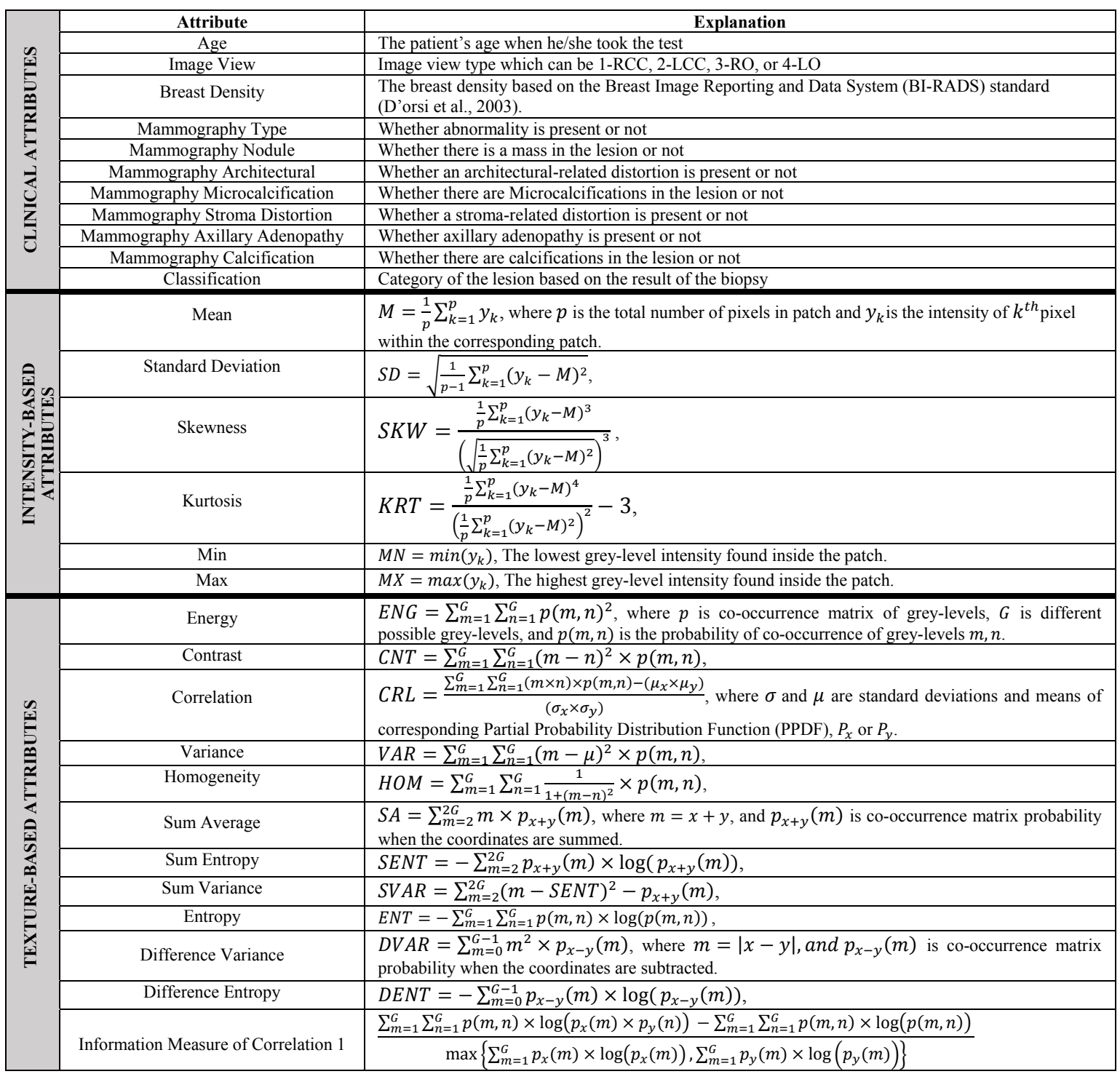




\begin{tabular}{|c|c|c|}
\hline & Information Measure of Correlation 2 & 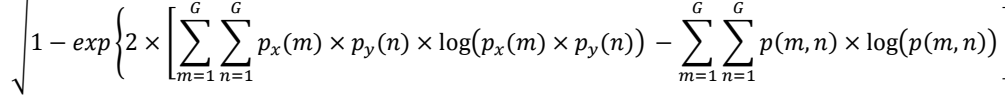 \\
\hline \multirow{8}{*}{ 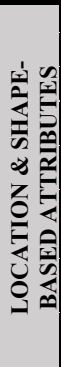 } & Area & $A=|p i x|$, where $p i x$ indicates the segmented area's pixels \\
\hline & Perimeter & Per $=\ln g(E)$, where $\ln g(E)$ indicates the length of edge pixels \\
\hline & Centre of Mass & COM $=$ coordinates of the mass centre \\
\hline & Solidity & $S l d=A /|C H|$, where $C H$ is the set of convex hull pixels of the patch \\
\hline & Extent & $E x t=A /|B B|$, where $B B$ is the set of bounding box pixels of the patch \\
\hline & Elongation & $\begin{array}{l}E l g=m n / m j \text {, where } m n \text { and } m j \text { are minor and major axes of most similar ellipse to the patch (having } \\
\text { the equal second central moments), respectively. }\end{array}$ \\
\hline & Circularity & $\operatorname{Crl}=4 \pi \times \frac{A}{\operatorname{Per}^{2}}$ \\
\hline & Form & Form $=\frac{\text { Elg } \times \text { Per }}{8 \times A}$, \\
\hline
\end{tabular}

Having these attributes, our aim is to select the most effective subset of attributes that renders the highest classification accuracy for early detection of breast cancer. This will be done through local and global search methods that are presented in the following section.

\section{THE PROPOSED ABCSA ALGORITHM}

Artificial Bee Colony (ABC) (Karaboga \& Basturk, 2007) is a swarm intelligence algorithm with good global exploration ability. Also, Simulated Annealing (SA) is a metaheuristic single-solution optimization algorithm which has a very good local search mechanism (Shokouhifar \& Jalali, 2015). Our motivation is to incorporate the advantages of both $\mathrm{ABC}$ and SA into a hybrid ABCSA algorithm. Generally, in the beginning of the optimization process, we search globally in the search space. On the other hand, local search can be very useful at the later iterations. In our approach, at first, $\mathrm{ABC}$ is performed, and then, $\mathrm{SA}$ is used to search in the vicinity of the best solution found by $\mathrm{ABC}$, in order to improve the best solution of ABC. The overall flowchart of the proposed hybrid ABCSA algorithm can be seen in Fig. 1.

\subsection{Problem Representation}

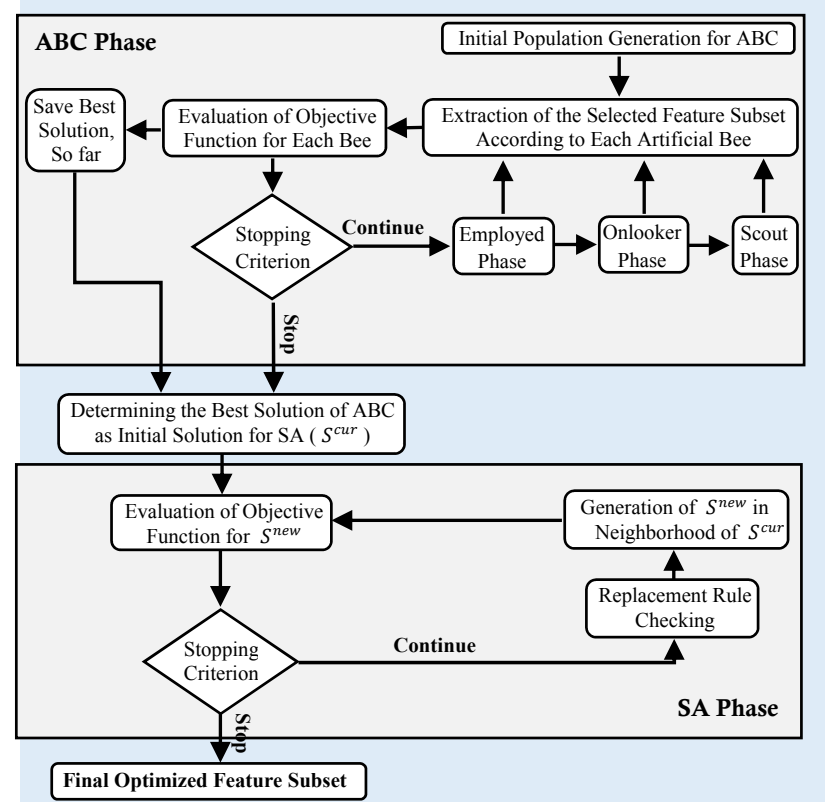

Figure 1. Overall flowchart of the ABCSA algorithm

In both $\mathrm{ABC}$ and SA phases, as seen in Fig. 2, a feasible solution can be represented as a binary string of length $L$, where $L$ is the total number of original features. The multi-objective cost function in both $\mathrm{ABC}$ and $\mathrm{SA}$ phases contains two objectives, both to be minimized. On the other hand, the first objective (the percent of the selected features) is in conflict with the second objective (the classification error), in such a way that as the first objective minimized (more discarded features), it would generate a greater classification error rate. In this case, the two objective functions can be combined into a single one by means of a weighted sum, as follows:

$$
\begin{gathered}
\text { Minimize }\left\{\text { Cost }=w_{1} \times\left(\frac{1}{L} \sum_{i=1}^{L} S_{i}\right)+w_{2} \times E\right\} \\
S_{i}= \begin{cases}1 & \text { if feature i has been selected } \\
0 & \text { otherwise }\end{cases}
\end{gathered}
$$

where the left term is to minimize the number of features, and the right term is to minimize the classification error achieved via SVM on the test instance set. $w_{1}$ and $w_{2}$ are two constant weights to adjust the relative importance of the two terms within the objective function. Since the classification accuracy is more important than the selected feature subset size, we assume $w_{2} \gg w_{1}$.

\begin{tabular}{|l|l|l|l|l|l|l|l|l|}
\hline \multicolumn{1}{l}{1} & 2 & 3 & 4 & 5 & 6 & 7 & & \multicolumn{1}{l}{} \\
\hline $\mathbf{0}$ & $\mathbf{1}$ & $\mathbf{0}$ & $\mathbf{0}$ & $\mathbf{1}$ & $\mathbf{0}$ & $\mathbf{1}$ & $\cdots$ & $\mathbf{1}$ \\
\hline
\end{tabular}

Figure 2. A feasible solution: "1" denotes that the corresponding feature is present, and " 0 " otherwise. 


\subsection{Global Search via $\mathbf{A B C}$}

In $\mathrm{ABC}$, each feasible solution corresponds to a food source position in $L$-dimension area (see Fig. 2), where $L$ is the number of optimization variables (number of original features). The nectar value of a feasible solution relates to the fitness of the solution. The nectar value (fitness) of $k^{\text {th }}$ bee is calculated as Fitness $_{k}=1 /$ Cost $_{k}$, in which, Cost $_{k}$ is the cost of $k^{\text {th }}$ bee to be minimized. An artificial bee colony consists of three kinds of artificial bees: employed bees, onlooker bees and scout bees (Shokouhifar \& Jalali, 2014). At first, the initial population is randomly generated. At the every iteration, each employed bee is moved onto her previously visited food source environment to explore a new food source in the vicinity of the present one. If the nectar amount of the new solution is higher, the bee forgets the previous one and memorizes the new one. The employed bee whose food source has been abandoned will become a scout bee. It is controlled by a parameter called limit. Scout bees carry out random searching to discover a new solution. Whenever all employed bees construct their solutions, they come back into the hive and share their information with onlookers. As seen in Eq. (3), the more nectar the employed contains, the more probability the onlooker to choose it, in which, $n$ is the number of employed bees, and $\beta$ is a constant parameter. Each onlooker goes onto the area of the selected employed bee, in order to explore a new food source within its vicinity.

\subsection{Local Search via SA}

$$
P_{k}=\frac{\left(\text { Fitness }_{k}\right)^{\beta}}{\sum_{k=1}^{n}\left(\text { Fitness }_{j}\right)^{\beta}}
$$

Generally, SA starts with a random initial solution. However, in this study, the final global best solution of $\mathrm{ABC}$ is selected as the initial solution for SA. The role of SA is to search in the vicinity of the final solution of $\mathrm{ABC}$, with the aim to improve it. At every iteration, a new solution $\left(S^{\text {new }}\right)$ is generated in the neighborhood of the current one $\left(S^{\text {cur }}\right)$. If $\operatorname{Cost}^{\text {new }}<\operatorname{Cost}^{\text {cur }}$, the current solution is replaced with the new one. If $\operatorname{Cost}^{\text {new }}>$ Cost ${ }^{\text {cur }}$, the new solution may be accepted with the probability of:

$$
P_{w}=\exp \left(-\left(\text { Cost }^{\text {new }}-\text { Cost }^{\text {cur }}\right) / T\right)
$$

where $\operatorname{Cost}^{\text {cur }}$ and $\operatorname{Cost}^{\text {new }}$ are the cost function for $S^{\text {cur }}$ and $S^{\text {new }}$, respectively. Also, $t_{S A}$ and max $_{-} i t e r_{S A}$ are the current iteration, and the defined number of iterations in SA, respectively. The temperature $T$ is considered to decrease linearly from $T_{\text {initial }}$ (initial temperature) to $T_{\text {final }}$ (final temperature), during algorithm execution, as follows:

$$
T=T_{\text {initial }}+\frac{t_{S A}}{\text { max_iter }_{S A}} \times\left(T_{\text {final }}-T_{\text {initial }}\right)
$$

As demonstrated in Fig. 3, binary swap operator is utilized for the neighborhood search in both $\mathrm{ABC}$ and SA phases.

\section{SIMULATION RESULTS}

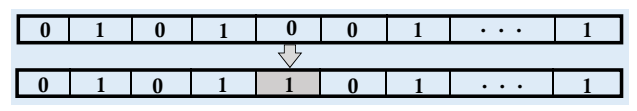

Figure 3. Binary swap operator for the neighborhood search in both $\mathrm{ABC}$ and $\mathrm{SA}$

In order to adjust the controllable parameters of the proposed ABCSA algorithm, different values were evaluated and the best ones (in terms of objective function and convergence speed) were determined. Settings for the parameters of the proposed hybrid ABCSA algorithm can be summarized in Table 4. Different kernel functions for SVM classifier are evaluated, and the linear kernel achieved the best result among them. Thus, we use linear kernel for the SVM classifier in our experiments. The obtained results of the ABCSA algorithm and comparison with the original datasets (containing all original features) can be summarized in Table 5 . Furthermore, we have compared the obtained results with other metaheuristic feature selection algorithms including Genetic Algorithm, Artificial Bee Colony, and Simulated Annealing.

Table 4. Settings for the parameters of the proposed ABCSA algorithm

\begin{tabular}{|l|l|}
\hline \multicolumn{1}{|c|}{ Parameter } & \multicolumn{1}{c|}{ Value/Description } \\
\hline max_iter $_{A B C}$ & $L$ \\
\hline Population size of ABC phase & 20 \\
\hline Number of employed bees & 25 (the half of colony) \\
\hline Number of onlooker bees & 25 (the half of colony) \\
\hline Limit & $\begin{array}{c}2.5 \text { order larger than the } \\
\text { best cost }\end{array}$ \\
\hline$\beta$ in Eq. (3) & 5 \\
\hline max_iter SA $_{\text {initial }}$ in Eq. $(5)$ & $2 \times L$ \\
\hline$T_{\text {final }}$ in Eq. $(5)$ & 0.01 \\
\hline$w_{1}$ in Eq. $(1)$ & 0 \\
\hline$w_{2}$ in Eq. $(1)$ & 0.01 \\
\hline & 0.99 \\
\hline
\end{tabular}

Table 5. Comparison of the results of the proposed ABCSA algorithm with original datasets

\begin{tabular}{|c|c|c|c|c|c|}
\hline \multicolumn{2}{|c|}{ Dataset Name } & \# Features & Sens & Spec & Acc \\
\hline \multirow{2}{*}{ SFM1 } & All Features & 38 & $81.03 \%$ & $88.24 \%$ & $84.4 \%$ \\
\cline { 2 - 6 } & Selected Features & 29 & $87.93 \%$ & $86.27 \%$ & $87.16 \%$ \\
\hline \multirow{2}{*}{ SFM2 } & All Features & 38 & $64.29 \%$ & $82.14 \%$ & $73.21 \%$ \\
\cline { 2 - 6 } & Selected Features & 19 & $78.57 \%$ & $89.29 \%$ & $83.93 \%$ \\
\hline \multirow{2}{*}{ FFDM1 } & All Features & 38 & $93.75 \%$ & $81.48 \%$ & $86.05 \%$ \\
\cline { 2 - 6 } & Selected Features & 8 & $93.75 \%$ & $96.3 \%$ & $95.35 \%$ \\
\hline \multirow{2}{*}{ FFDM2 } & All Features & 38 & $93.33 \%$ & $99.17 \%$ & $98.53 \%$ \\
\cline { 2 - 6 } & Selected Features & 23 & $100 \%$ & $100 \%$ & $100 \%$ \\
\hline
\end{tabular}


The simulation results can be seen in Table 6, in terms of the number of selected features and final accuracy. According to result of the simulations, the proposed ABCSA outperforms the other metaheuristic algorithms, in terms of the minimization of the selected features while maximizing the detection accuracy. From Table 6 , it can be seen that the proposed ABCSA algorithm outperforms all compared algorithms for the two datasets SFM1 and FFDM1. In the case of SFM2 dataset, the ABCSA and ABC have achieved equal accuracy, and the both algorithms outperform GA and SA. However, ABCSA has 19 features (3 features lower than ABC). Finally, in the case of FFDM2 dataset, the proposed ABCSA, ABC and GA have achieved the maximum accuracy $100 \%$. In this case, both ABCSA and ABC have 23 features. As seen, in SFM1 and FFDM1 ABCSA outperforms $\mathrm{ABC}$, but in SFM2 and FFDM2, both ABCSA and $\mathrm{ABC}$ have equal accuracy. It is due to added local search exploration via SA. On the other hand, in SFM1 and FFDM1 datasets, the added local search mechanism (via SA) leads to improve the result of $\mathrm{ABC}$.

Table 6. Comparison of the results of the ABCSA with other metaheuristic feature selection algorithms

\begin{tabular}{|c|c|c|c|c|c|c|c|c|}
\hline \multirow{2}{*}{ Dataset Name } & \multicolumn{2}{|c|}{ GA } & \multicolumn{2}{|c|}{ SA } & \multicolumn{3}{c|}{ ABC } & \multicolumn{2}{c|}{ Proposed (ABCSA) } \\
\cline { 2 - 8 } & \# Features & Accuracy & \# Features & Accuracy & \# Features & Accuracy & \# Features & Accuracy \\
\hline SFM1 & 27 & $86.32 \%$ & 32 & $84.91 \%$ & 24 & $85.54 \%$ & 29 & $87.16 \%$ \\
\hline SFM2 & 16 & $79.19 \%$ & 26 & $80.6 \%$ & 22 & $83.93 \%$ & 19 & $83.93 \%$ \\
\hline FFDM1 & 15 & $92.26 \%$ & 17 & $94.12 \%$ & 13 & $93.33 \%$ & 8 & $95.35 \%$ \\
\hline FFDM2 & 26 & $100 \%$ & 19 & $99.24 \%$ & 23 & $100 \%$ & 23 & $100 \%$ \\
\hline
\end{tabular}

\section{DISCUSSION AND CONCLUSIONS}

The best and most common way of breast cancer diagnosis is mammography which has been reported to be able to detect the cancer up to four years in advance. Therefore, in this paper, a hybrid feature selection algorithm based on both local and global search paradigms has been presented for the early detection of breast cancer based on two types of mammogram images. The central objective of this paper is to apply ABC (as global search method) and SA (as local search method) to find the best subset of features in order to accurately classify benign and healthy patients. The proposed hybrid algorithm is called ABCSA. We have used SVM as the classifier. Simulations show that the proposed ABCSA algorithm outperforms the existing metaheuristic feature selection algorithms in terms of maximizing the detection accuracy. As a future work, we plan to utilize the proposed methodology for genome-based breast cancer datasets. We also plan to propose other hybrid global and local search algorithms for effective feature selection in different problems.

\section{REFERENCES}

Abroudi, A., Shokouhifar, M., \& Farokhi, F. (2013). Improving the Performance of Artificial Neural Networks via Instance Selection and Feature Dimensionality Reduction. Int. J. Mach. Learn. Comput, 2, 176180.

Al Mutaz, M. A., Dress, S., \& Zaki, N. (2011). Detection of masses in digital mammogram using second order statistics and artificial neural network. International Journal of Computer Science \& Information Technology (IJCSIT), 3(3), 176-186.

Baeg, S., \& Kehtarnavaz, N. (2002). Classification of breast mass abnormalities using denseness and architectural distortion. Electronic Letters on Computer Vision and Image Analysis, 1(1), 1-20.

Bellotti, R., De Carlo, F., Tangaro, S., Gargano, G., Maggipinto, G., Castellano, M., . . Magro, R. (2006). A completely automated CAD system for mass detection in a large mammographic database. Medical physics, 33(8), 3066-3075.

Brem, R. F., Baum, J., Lechner, M., Kaplan, S., Souders, S., Naul, L. G., \& Hoffmeister, J. (2003). Improvement in sensitivity of screening mammography with computer-aided detection: a multiinstitutional trial. American Journal of Roentgenology, 181(3), 687-693.

Bruce, L. M., \& Adhami, R. R. (1999). Classifying mammographic mass shapes using the wavelet transform modulus-maxima method. Medical Imaging, IEEE Transactions on, 18(12), 1170-1177.

Campanini, R., Dongiovanni, D., Iampieri, E., Lanconelli, N., Masotti, M., Palermo, G., . . Roffilli, M. (2004). A novel featureless approach to mass detection in digital mammograms based on support vector machines. Physics in Medicine and Biology, 49(6), 961.

Chan, H.-P., Wei, D., Helvie, M. A., Sahiner, B., Adler, D. D., Goodsitt, M. M., \& Petrick, N. (1995). Computer-aided classification of mammographic masses and normal tissue: linear discriminant analysis in texture feature space. Physics in medicine and biology, 40(5), 857.

D’orsi, C., Bassett, L., Berg, W., Feig, S., Jackson, V., \& Kopans, D. (2003). Breast imaging reporting and data system: ACR BI-RADS-mammography. American College of Radiology, 4.

De Santo, M., Molinara, M., Tortorella, F., \& Vento, M. (2003). Automatic classification of clustered microcalcifications by a multiple expert system. Pattern Recognition, 36(7), 1467-1477. 
Dinesh, M. (2011). Model based approach for detection of architectural distortions and spiculated masses in mammograms.

Faridah, Y. (2008). Digital versus screen film mammography: a clinical comparison. Biomedical imaging and intervention journal, 4(4), e31-e31.

Guo, Q., Shao, J., \& Ruiz, V. (2005). Investigation of support vector machine for the detection of architectural distortion in mammographic imagesIOP Publishing. Symposium conducted at the meeting of the Journal of Physics: Conference Series

Hadjiiski, L., Sahiner, B., Chan, H.-P., Petrick, N., \& Helvie, M. (1999). Classification of malignant and benign masses based on hybrid ART2LDA approach. Medical Imaging, IEEE Transactions on, 18(12), 11781187.

Karaboga, D., \& Basturk, B. (2007). A powerful and efficient algorithm for numerical function optimization: artificial bee colony (ABC) algorithm. Journal of global optimization, 39(3), 459-471.

Kinoshita, S., Marques, P. A., Slaets, A., Marana, H., Ferrari, R., \& Villela, R. (1998). Detection and characterization of mammographic masses by artificial neural network. In Digital Mammography (pp. 489-490): Springer.

López, M. A. G., de Posada, N. G., Moura, D. C., Pollán, R. R., Valiente, J. M. F., Ortega, C. S., .. Loureiro10, J. P. (2012). BCDR: A BREAST CANCER DIGITAL REPOSITORY Symposium conducted at the meeting of the 15th International Conference on Experimental Mechanics

Moura, D. C., \& López, M. A. G. (2013). An evaluation of image descriptors combined with clinical data for breast cancer diagnosis. International journal of computer assisted radiology and surgery, 8(4), 561574.

Moura, D. C., López, M. A. G., Pollan, R. R., Ramos, I. M. P., Loureiro, J. P., Fernandes, T. C., \& de Araújo, B. M. F. (2012). Classifier Performance Vs Dataset Size: A Comparative Study For Breast Lesions Classification Symposium conducted at the meeting of the 15th International Conference on Experimental Mechanics (22-17 July 2012), Porto, Portugal

Mudigonda, N. R., Rangayyan, R. M., \& Desautels, J. L. (2001). Detection of breast masses in mammograms by density slicing and texture flow-field analysis. Medical Imaging, IEEE Transactions on, 20(12), 1215-1227.

Pisano, E. D., Gatsonis, C., Hendrick, E., Yaffe, M., Baum, J. K., Acharyya, S., . . . D'Orsi, C. (2005). Diagnostic performance of digital versus film mammography for breast-cancer screening. New England Journal of Medicine, 353(17), 1773-1783.

Pisano, E. D., Hendrick, R. E., Yaffe, M. J., Baum, J. K., Acharyya, S., Cormack, J. B., . . . Bassett, L. W. (2008). Diagnostic Accuracy of Digital versus Film Mammography: Exploratory Analysis of Selected Population Subgroups in DMIST 1. Radiology, 246(2), 376-383.

Sahiner, B., Chan, H.-P., Petrick, N., Wei, D., Helvie, M. A., Adler, D. D., \& Goodsitt, M. M. (1996). Classification of mass and normal breast tissue: a convolution neural network classifier with spatial domain and texture images. Medical Imaging, IEEE Transactions on, 15(5), 598-610.

Sampat, M. P., Markey, M. K., \& Bovik, A. C. (2005). Computer-aided detection and diagnosis in mammography. Handbook of image and video processing, 2(1), 1195-1217.

Shokouhifar, M., \& Jalali, A. (2014). Real-time task scheduling in heterogeneous multiprocessor systems using artificial bee colonyIEEE. Symposium conducted at the meeting of the Electrical Engineering (ICEE), 2014 22nd Iranian Conference on

Shokouhifar, M., \& Jalali, A. (2015). An evolutionary-based methodology for symbolic simplification of analog circuits using genetic algorithm and simulated annealing. Expert Systems with Applications, 42(3), 1189-1201.

Spurgeon, D. (2005). Digital mammography is more accurate only for certain groups of women. BMJ: British Medical Journal, 331(7518), 653.

Timp, S., Varela, C., \& Karssemeijer, N. (2007). Temporal change analysis for characterization of mass lesions in mammography. Medical Imaging, IEEE Transactions on, 26(7), 945-953.

Torre, L. A., Bray, F., Siegel, R. L., Ferlay, J., Lortet-Tieulent, J., \& Jemal, A. (2015). Global cancer statistics, 2012. CA: a cancer journal for clinicians, 65(2), 87-108.

Verma, B., \& Zakos, J. (2001). A computer-aided diagnosis system for digital mammograms based on fuzzyneural and feature extraction techniques. Information Technology in Biomedicine, IEEE Transactions on, 5(1), 46-54. 